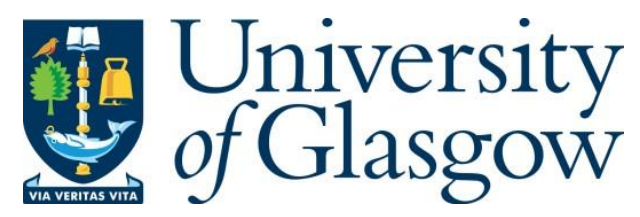

Asad, S. M., Ozturk, M., Rais, R. N. B., Zoha, A., Hussain, S., Abbasi, Q. H. and Imran, M. A. (2019) Reinforcement Learning Driven Energy Efficient Mobile Communication and Applications. In: 2019 IEEE International Symposium on Signal Processing and Information Technology (ISSPIT), Ajman, United Arab Emirates, 10-12 Dec 2019, ISBN 9781728153414.

There may be differences between this version and the published version. You are advised to consult the publisher's version if you wish to cite from it.

http://eprints.gla.ac.uk/202348/

Deposited on: 4 November 2019

Enlighten - Research publications by members of the University of Glasgow http://eprints.gla.ac.uk 


\title{
Reinforcement Learning driven Energy Efficient Mobile Communication and Applications
}

\author{
Syed Muhammad Asad*, Metin Ozturk*, Rao Naveed Bin Rais ${ }^{\dagger}$, Ahmed Zoha*, Sajjad Hussain* \\ Qammer H. Abbasi*, Muhammad Ali Imran* \\ * James Watt School of Engineering, University of Glasgow, Glasgow, G12 8QQ, UK \\ s.asad.1@research@gla.ac.uk, m.ozturk.1@research.gla.ac.uk, \\ \{Ahmed.Zoha, Sajjad. Hussain, Qammer.Abbasi, Muhammad.Imran\}@glasgow.ac.uk, \\ $\dagger$ Electrical and Computer Engineering, Ajman University, UAE \\ r.rais@ajman.ac.ae
}

\begin{abstract}
Smart city planning is envisaged as advance technology based independent and autonomous environment enabled by optimal utilisation of resources to meet the short and long run needs of its citizens. It is therefore, preeminent area of research to improve the energy consumption as a potential solution in multi-tier 5G Heterogeneous Networks (HetNets). This article predominantly focuses on energy consumption coupled with $\mathrm{CO}_{2}$ emissions in cellular networks in the context of smart cities. We use Reinforcement Learning (RL) vertical traffic offloading algorithm to optimize energy consumption in Base Stations (BSs) and to reduce carbon footprint by applying widely accepted strategy of cell switching and traffic offloading. The algorithm relies on a macro cell and multiple small cells traffic load information to determine the cell offloading strategy in most energy efficient way while maintaining quality of service demands and fulfilling users applications. Spatio-temporal simulations are performed to determine a cell switch on/off operation and offload strategy using varying traffic conditions in control data separated architecture. The simulation results of the proposed scheme prove to achieve reasonable percentage of energy and $\mathrm{CO}_{2}$ reduction.

Index Terms-Smart City Planning, Green Communications, Energy Efficiency, Vertical Offloading, Machine Learning, 5G.
\end{abstract}

\section{INTRODUCTION}

Mobile Communication is responsible for $2 \%$ of global $\mathrm{CO}_{2}$ emissions with the potential to increase to approximately $4 \%$ by 2020 [1], [2] where data is in high demands likely to increase manifold. This would result in potential rise in energy consumption. To mitigate the impacts on the environment with such increased energy consumption, cell switching and traffic offloading is required in an effective manner which would have direct impact on overall operational expenditure, cell power and energy consumptions, and $\mathrm{CO}_{2}$ emissions.

Nowadays, with the increased demands of mobile communications and its applications that lead to a number of mobile subscribers continue to grow with high data traffic demands. The problem is manifold by the limited amount of available resources in cellular networks [3]. Therefore, traditional Macro Base Stations (MBSs) encounter several challenges to offer high data rates in highly dense environment. As this has been brought into various discussions that a MBS has limited mobile network channels offered by regulatory authority to transmit on a limited scale to serve number of users [4]. Similarly, with the increase in the deployment of
Small Cells (SCs), energy consumption dramatically increases which brings challenge to mobile network operators when dimensioning their network in order to control cost and support smart city planning and green communications agenda ${ }^{1}$. These challenges lead to a conclusion discussed in many literatures such as [5], that traditional Macro Cells (MCs) with large coverage footprints would be broken into multiple SCs.

A logical separation between MBS and Data Base Stations (DBSs) is determined by control data separated architecture (CDSA) where control and data planes are separated [6]. The key concept behind this approach is to separate signalling function required to ensure coverage from those needed to support high data rate transmissions and to take the advantage of spatial reuse. In this Radio Access Network (RAN) architecture, MBS are dedicated to provide signalling and support efficient Radio Resource Control (RRC) procedures whereas DBSs are responsible for high data rate transmissions. The proposed approach provides stringent measure to meet high data traffic demands and maintain Quality of Service (QoS) within set boundaries of regulatory authorities. Such architecture is heralded as most promising way to increase coverage and capacity in efficient manner as defined in [6]. However, withe the growing number of BSs has a direct impact on increased energy consumption and $\mathrm{CO}_{2}$ emissions.

In order to maintain QoS, there are three offloading schemes discussed in the literature which are vertical, horizontal and joint traffic offloading [7], [8]. Vertical traffic offloading shifts the SC load to MC whereas horizontal traffic offloading offload the SC traffic to a neighbouring SC. In Joint traffic offloading, both vertical and horizontal schemes are used. Some literatures considered use of RL in order to switch cells and offload traffic such as [9].

There are many literature such as [10], [11] which discussed the concept that Energy Efficiency (EE) of the network can be improved by traffic offloading and cell on/off switching method, but none of them calculated the impact of energy on $\mathrm{CO}_{2}$ emissions. In this paper, our focus is to determine energy aware methodology and its impact on $\mathrm{CO}_{2}$ emissions of the

\footnotetext{
${ }^{1}$ Mayor of London Transport Strategy can be found online at: https://www.london.gov.uk/sites/default/files/mayorstransport-strategy2018.pdf.
} 


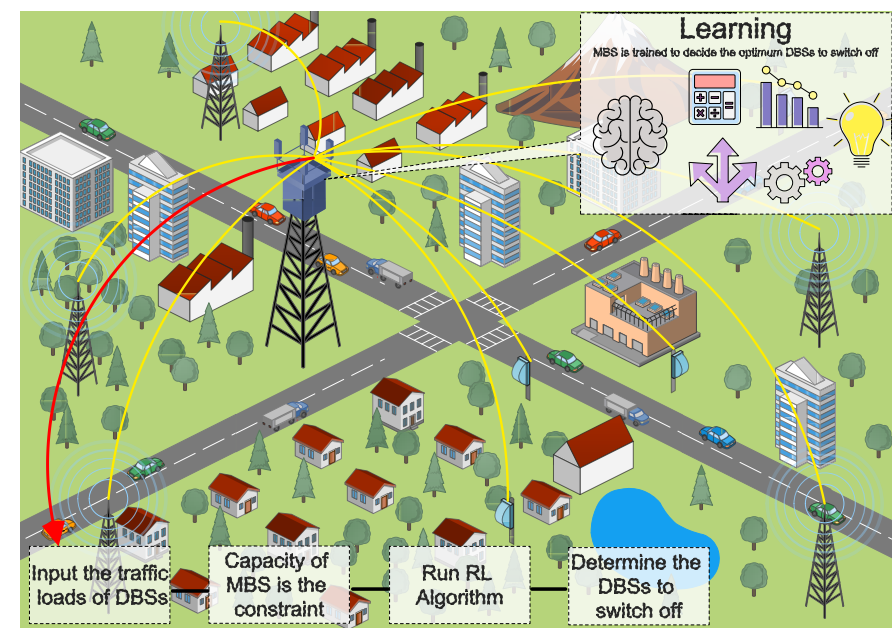

Fig. 1. HetNet Architecture with SCs uniformly distributed around MC.

entire HetNet model which comprises of a MC and multiple SCs by using RL vertical offloading method. Finally, we compare the impact of such approach against overall energy consumption and reduced $\mathrm{CO}_{2}$ emissions.

Our major contribution here, is RL based novel cell switching (CS) scheme dependant on BS static and dynamic load profiles considering live BSs in the dense city environment to establish carbon footprint reduction associated with BS energy consumption. Real traffic and user mobility data have been obtained by Mobile Network Operators (MNOs) in the UK along with location of operational SCs in the city of London to verify the proposed approach.

\section{SySTEM MODEL}

\section{A. HetNet Architecture}

An approach to densify the network where multiple SCs are deployed under one $\mathrm{MC}$ footprint has been proven an effective method to improve capacity. A holistic view on ultra-dense SC and HetNets is presented in [11]. This results, with the small coverage radius compared to conventional MC where SCs transmission power is reduced which eventually enhances capacity, reduces cost and improves EE of the network. In order to analyse HetNet energy performance and its impact on $\mathrm{CO}_{2}$ emissions, a multi-tier cellular network comprises of a MC and multiple SCs that are surrounded by MC under its coverage foot print is shown in Fig. 1. However, with the discussed approach, several technical challenges start to occur which includes unpremeditated deployment, intercell interference, non-seamless handovers, back-haul overload and inefficient energy consumption.

Our main goal, as a first step, is to design a wireless network to derive overall energy consumption, therefore twotier HetNet model is considered. The MC is used to provide low data rate services, continuous coverage and signalling in its footprint. Whereas, the SCs are responsible to provide high capacity data rates serving their users within their coverage footprint. All SCs are connected to MC by a back-haul link.
TABLE I

POWER CONSUMPTION OF A TYPICAL BS

\begin{tabular}{lll}
\hline Equipment & Abbreviation & Value \\
\hline Power amplifier (MIMO) & $B S_{\mathrm{amp}}$ & $600 \mathrm{~W}$ \\
Power amplifier efficiency & $P A_{\mathrm{eff}}$ & $10 \%$ \\
Antenna input power (MIMO) & $A_{\mathrm{i}}$ & $40 \mathrm{~W}$ \\
Transceiver & $P r_{\mathrm{t}}$ & $100 \mathrm{~W}$ \\
Digital signal processor & $P r_{\mathrm{d}}$ & $100 \mathrm{~W}$ \\
Signal generator & $P r_{\mathrm{g}}$ & $400 \mathrm{~W}$ \\
AC-DC converter & $P r_{\mathrm{c}}$ & $100 \mathrm{~W}$ \\
Back-haul link & $P r_{\mathrm{l}}$ & $100 \mathrm{~W}$ \\
Others & $P r_{\mathrm{o}}$ & $100 \mathrm{~W}$ \\
\hline
\end{tabular}

RL algorithm driven by vertical offloading, monitors low traffic activity where it switches off the lightly loaded SCs and offload its traffic to MC. Vertical offloading, is a technique to provide continuous service across all SCs within HetNet where user does not experience any transference of services during the offloading procedure. In order to reduce energy, vertical offloading plays a vital role when users are seamlessly migrated to MC. Overlapping between the SCs can happen provided the total sum of their areas do not exceed the MC coverage radius. Finally, $\mathrm{CO}_{2}$ emissions are analysed for the proposed cell switching and traffic offloading approach.

\section{B. Energy Consumption Model}

For wireless network performance evaluation, the broadly accepted state of the art is to analyse components of RAN at system level. There are multiple components in a typical BS that contributes to certain level of power consumption depend on traffic load profiles. These components include, power amplifiers, back-haul links, amplifier efficiency, signal processing and generation, air conditioning and others. The power consumption of typical BS components is summarised in Table I.

In order to determine the total power consumption by a typical BS with all of its components is:

$$
\begin{aligned}
& B S_{\mathrm{tot}}=\begin{array}{c}
S\left[\left(A_{\mathrm{Tx}} B S_{\mathrm{amp}}\right)+P r_{\mathrm{t}}+P r_{\mathrm{d}}+\right. \\
\left.P r_{\mathrm{g}}+P r_{\mathrm{c}}+P r_{\mathrm{o}}\right]+P r_{1}+P r_{\mathrm{a}},
\end{array} \\
& B S_{\text {amp }}=\frac{A_{\mathrm{i}}}{P A_{\mathrm{eff}}},
\end{aligned}
$$

where $S$ is the number of sectors in a cell, $A_{\mathrm{Tx}}$ is the number of antennas transmitting per sector. The power consumption of a typical BS components are represented by; power amplifier as $B S_{\text {amp }}$, transceiver as $P r_{\mathrm{t}}$, digital signal processor as $P r_{\mathrm{d}}$, signal generator as $P r_{\mathrm{g}}$, AC-DC converter as $P r_{\mathrm{c}}$, back-haul link as $P r_{1}$, air conditioning as $P r_{\text {a }}$ respectively. There may be other components which contribute to the total BS power consumption are termed as $\operatorname{Pr}_{\mathrm{o}}$. We can calculate the total power being consumed by HetNet as: 


$$
P_{\mathrm{HetNet}}=P_{\mathrm{mc}}+\sum_{k=2}^{K} P_{\mathrm{sc}}^{k},
$$

where $P_{\text {HetNet }}$ is total HetNet power consumption, $P_{\mathrm{mc}}$ and $P_{\mathrm{sc}}^{k}$ are the power consumptions of $\mathrm{MC}$ and $\mathrm{K}$-th SCs respectively. The total power consumption of a MC would be expressed as:

$$
P_{\mathrm{mc}}=B S_{\mathrm{tot}}^{\mathrm{mc}}+\delta_{\mathrm{mc}} P_{\mathrm{tx}}^{\mathrm{mc}},
$$

where $P_{\mathrm{mc}}$ is the total power consumption of a $\mathrm{MC}, B S_{\mathrm{tot}}^{\mathrm{mc}}$ is the power calculated in (1) for $\mathrm{MC}, \delta_{\mathrm{mc}}$ is the component which has dependency on load profile of $\mathrm{MC}$ and $P_{\mathrm{tx}}^{\mathrm{sc}}$ is the load of the MC per 15 minutes. Similarly, the total power consumption of a $\mathrm{SC}$ can be calculated as:

$$
P_{\mathrm{sc}}^{k}=B S_{\mathrm{tot}}^{\mathrm{sc}}+\delta_{\mathrm{sc}} P_{\mathrm{tx}}^{\mathrm{sc}},
$$

where $P_{\mathrm{sc}}^{\mathrm{k}}$ denotes the total SC power consumption, $k=$ $\{2,3,4, . ., n\}$, is the number of SCs surrounded by a MC, $B S_{\text {tot }}^{\text {sc }}$ is the power calculated in (1) for each of the individual SCs, $\delta_{\text {sc }}$ is the load dependent component of power consumption of the SC and $P_{\mathrm{tx}}^{\mathrm{sc}}$ represents the load of the SC per 15 minutes. Therefore, from (3), the total energy consumption $E_{\mathrm{HetNet}}$ for each time interval $t$ would be determined.

In order to assess entire network EE performance for each time interval, a ratio of expected capacity consumed by HetNet $C_{\mathrm{m}}$ to the maximum HetNet power consumption $P_{\text {HetNet }}$ needs to be calculated with the following:

$$
E E_{\text {tot }}=\frac{C_{\mathrm{m}}}{P_{\mathrm{HetNet}}},
$$

\section{Cell Load}

There is rich literature already been presented in many papers on Handover (HO) decision algorithms for small cells, e.g. [12] that incorporates several radio parameters such as channel capacity, signal strength, signal quality, speed, and transmit power. As we know Shannon capacity is expressed by $C=B W \cdot \log (1+S I N R)$ where $C$ represents capacity associated with the channel bandwidth $B$ and Signal to Interference and Noise Ratio (SINR), we propose TO based on cell load profile associated with expected capacity of MC $E\left(C_{\mathrm{mc}}\right)$ and SCs $E\left(C_{\mathrm{sc}}\right)$ every 15 minutes over 24 hours duration $t$.

$$
C_{\mathrm{m}}=\lim _{t \rightarrow 24} \frac{E\left(C_{\mathrm{mc}}\right) t_{\mathrm{mc}}+E\left(C_{\mathrm{sc}}\right) t_{\mathrm{sc}}}{t},
$$

where $C_{\mathrm{m}}$ would be measured capacity, $t_{\mathrm{mc}}$ and $t_{\mathrm{sc}}$ represent the time of user association with MC and SC. In order to calculate expected capacities of MC and SC, we have (8), (9) where $x$ denotes SINR of the BSs in the HetNet:

$$
\begin{gathered}
E\left(C_{\mathrm{mc}}\right)=B W \int_{0}^{n} \log (1+x) d x, \\
E\left(C_{\mathrm{sc}}\right)=B W \sum_{i=2}^{N} \int_{0}^{n} \log \left(1+x_{\mathrm{i}}\right) d x,
\end{gathered}
$$

Therefore, the cell load $C L$ of $\mathrm{MC}$ and SCs is the ratio of measured $C_{\mathrm{m}}$ to $C_{\max }$ maximum capacity which is represented as $C L(\%)=C_{\mathrm{m}} / C_{\max }$. Thus, by normalising $C L$, load factor $\rho_{\mathrm{i}}$ is achieved. From the following equation, we can calculate transmitted power $P_{\mathrm{tx}}$ as:

$$
P_{\mathrm{tx}}=\rho_{i} P_{\max }
$$

where $P_{\max }$ is the maximum power output power of a BS and $i=\{1,15,30, \ldots, \mathrm{n}\}$ represented in minutes.

States of SCs when they switch on/off depend on the number of factors such as distance of a user from associated SC, user's movement out of the SC's radius range, load of the $\mathrm{SC}$ and time of the day (peak and off-peak hours). This can be represented as:

$$
\begin{cases}\delta_{0}, & t>T_{t h}, \rho<\rho_{t h}, d_{i}>R_{i}, \\ \delta_{1}, & t<T_{t h}, \rho>\rho_{t h}, d_{i}<R_{i},\end{cases}
$$

where, $\delta_{0}$ and $\delta_{1}$ denotes the two states at which a SC is off and on, $t$ is the time when SC is switch on/off depends on the threshold time $T_{t h}, \rho$ is the load factor of a SC when SC decides to switch on/off depending on threshold value of the load profile represented as $\rho_{t h}, d_{i}$ is distance of a user from $\mathrm{BS}$ and $R i$ represents $\mathrm{BS}$ radius.

\section{Carbon Emissions}

Use of carbon footprint ( $\mathrm{CO}_{2}$ emissions) is based on total energy of HetNet and can be calculated with the help of conversion factor described in [1], [13]. Therefore, from (3) we have;

$$
\Delta_{C O_{2}}=\psi \int_{0}^{T} E_{\mathrm{HetNet}}\left(\frac{P_{\mathrm{mc}}}{t}, \frac{P_{\mathrm{sc}}}{t}\right) d t,
$$

where $\Delta_{\mathrm{CO}_{2}}$ is carbon footprint associated with total energy consumption $E_{\mathrm{HetNet}}, \psi$ refers to emissions per unit/conversion factor and $t$ represents the time duration in which $E_{\text {HetNet }}$ has been calculated.

\section{Proposed Methodology}

Reinforcement learning driven vertical offloading method proposed in this work uses $Q$-Learning (QL) algorithm for sequential decision making variant on cell load conditions. We analysed the maximum ratio of under-loaded SCs in the time domain where users within the lightly loaded SCs are only offloaded to other MC called vertical offloading. Due to the low transmit powers of SCs in horizontal offloading and have limitations to a certain range, horizontal offloading can not always be realised between SCs. Therefore, for some SCs to go into the sleep mode when their neighbouring SCs are not in proximity, vertical offloading becomes the only choice. Action to offload traffic is taken when agent's collected information triggers under-loading situation. This action would be rewarded or penalised based on particular conditional state in a given time period.

QL algorithm is a form of RL which is model-free. In other words, it a method of asynchronous dynamic programming 
where it provides agents with the opportunity of learning that finds an estimate of the optimal action-value function by experiencing concurrent sequences of actions [14].

Since, RL would be able to handle wide range of tasks associated with actions, we have chosen RL algorithm where the MC interacts with the network environment, collects live user traffic information, compare the information with operational SCs energy consumption levels and their operational load through its back-haul connectivity. After learning from the network environment, MC takes decision whether or which $\mathrm{SC}$ are required to be switched off at a given period of time when they are either idle or lightly loaded. Hence, RL would be able to tackle with the challenging environment because it can adapt to changing needs driven by actions through continuous learning. QL algorithm has also proven capability of interacting in dynamic environments [10] with the six main components as (i) agent, (ii) environment, (iii) action, (iv) state, (v) reward/penalty, and (vi) action-value table. Agent's actions are environment dependant to maximise the reward or minimize the penalty. After the execution of each agent's action, resulting state and reward/penalty are evaluated. Following rule is applied once all the executions are completed:

$$
\begin{aligned}
Q\left(s_{t}, a_{t}\right) & :=Q\left(s_{t}, a_{t}\right)+ \\
& \phi\left[\gamma_{t+1}+\lambda \min _{a}\left(Q\left(s_{t+1}, a\right)\right)-Q\left(s_{t}, a_{t}\right)\right],
\end{aligned}
$$

where $s_{t}$ and $s_{t+1}$ are the current and next states, $\phi$ is a discount factor, $\gamma_{t+1}$ is the expected penalty for the next step and $a_{t}$ is the action taken after MC learned from the environment, $a$ is the set of all possible actions and $\lambda$ is the learning rate.

QL is an off-policy and model-free algorithm which follows different policies in determining the next actions and updates the action-value table where agent does not have knowledge of prior actions being taken in the environment, instead it take actions to obtain environment information. Due to its low computational overhead for BS switching QL algorithm proved to be the most chosen solution [14].

Our work comprises of $1 \mathrm{MC}$ and 9 SCs such that the state space in the $Q$ table is updated for every action-value pair. In different time intervals, $\mathrm{MC}$ obtains and records varying traffic condition of SCs in order to make decisions and eventually select set of SCs that are needed to be switched off.

The MC state space has dependency on availability of capacity and resources when it performs traffic monitoring, offloading and switching. The two possible states, $\delta_{1}$ and $\delta_{2}$, are described as follows:

$$
\begin{cases}\delta_{1}, & M_{c}, R_{m}<O_{c}, \\ \delta_{2}, & M_{c}, R_{m} \geqslant O_{c},\end{cases}
$$

where $M_{c}$ is the monitoring capacity, $R_{m}$ being the resources available after the capacity has been monitored. $O_{c}$ is the capacity of offloading. First state $\delta_{1}$ signifies the constraint when capacity of monitoring and available resources are not satisfied whereas $\delta_{2}$ satisfies the case. Now the total power consumption $\gamma$ of the network in (3) can be represented as:

$$
\gamma(a)=P_{\mathrm{HetNet}}\left(P_{\mathrm{mc}}, P_{\mathrm{sc}}^{\mathrm{k}}\right) .
$$

Therefore, based on power consumption of the BS(s), total energy consumption is calculated in each time interval. Finally, the use of $\mathrm{CO}_{2}$ emissions would be determined by using (12)

\section{Performance Evaluation}

\section{A. Data Set}

This section describes the distribution of users within each cell (either MC or SC) that are used to produce expected capacity over time in HetNet architecture. The number of active users in each cell varies over time in a day such that they are distributed over quarter intervals within an hour to form 24-hour duration. More specifically, in 24-hour duration, we have modelled 21-hours from 05:00 am to 02:00 am because of negligible traffic recorded in the remaining hours of night. The cell load then normalised to produce load factor $\rho_{\mathrm{i}}$ in order to calculate transmitted power $P_{\mathrm{tx}}$ from (10). Typical BS static power consumption is calculated with the help of multiple BS components as mentioned in (1). Therefore, by using BS static power, BS transmitted power and dependant load component, total power consumptions of all cells from (4), (5) are calculated. From 100 iterations, we plotted average mean of calculated energy consumptions for all cells with the gain percentages as shown in Fig. 2. The overall EE from (6) has also been plotted after running 100 iterations and averaging the values. Measured capacity for each BS in a specific time frame (15 minutes intervals) is divided by power consumption of the associated BS. The plot is shown in Fig. 3 where we have assumed $50 \%$ of the subscribers are heavy data users with average data rate of $2 \mathrm{Mb} / \mathrm{s}$ multiplied by number of users in each interval. There are many ways to calculate user demands by adjusting the ratio of low, medium and heavy users. However, our main focus is on $\mathrm{CO}_{2}$ emissions, therefore we have shown $\mathrm{EE}$ graphs to determine the relation of overall $\mathrm{EE}$ with our proposed methods. Finally, $\mathrm{CO}_{2}$ emissions associated with energy consumptions are presented in Fig. 4. Simulation parameters are mentioned in Table II [10], [15].

\section{B. Benchmarking}

In addition to the proposed $Q$-learning based CS approach, three more techniques are also developed to compare and assess the performance of the proposed method. Note that the $\mathrm{MC}$ is always on for all the methods that will be explained in the next paragraphs.

a) All-On: In this CS method, all the SCs are always kept on, meaning that no switching is implemented. Having this method in the results is quite important, since it is currently the case for the majority of the networks. Even though this method does not offer any saving in power consumption and/or $\mathrm{CO}_{2}$ emission, it does not suffer from reduced quality of service (QoS) given that all the users are kept connected with their best serving BS due to the fact that there is no switching and offloading. 


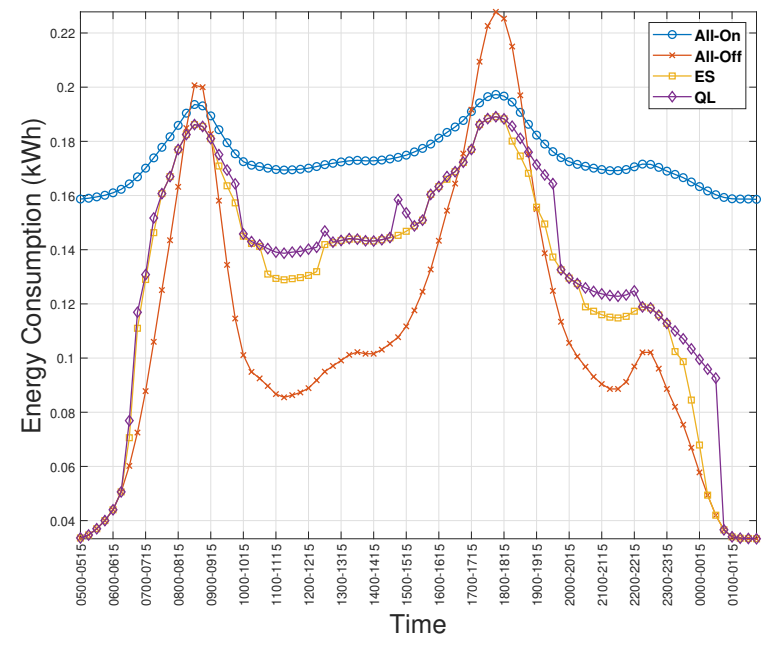

(a) Total energy consumption of the HetNet with 1 MC and 9 SCs.

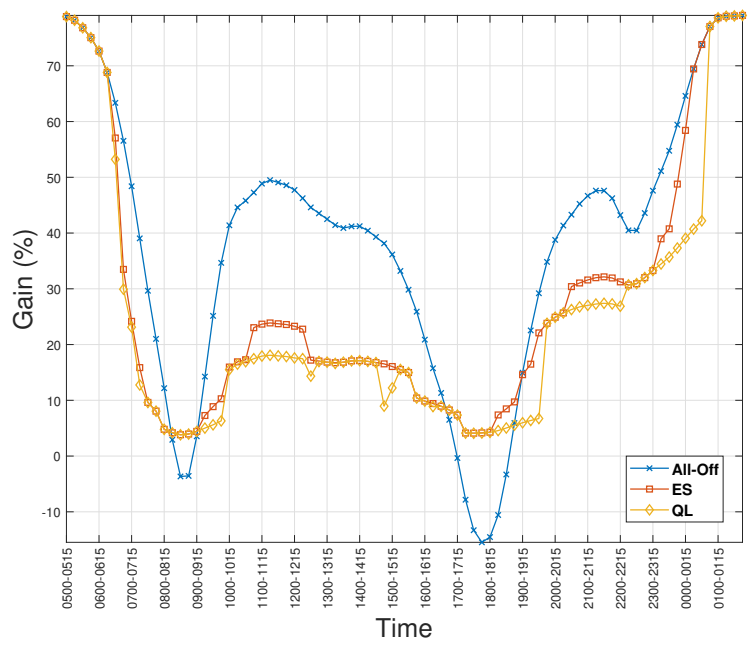

(b) Gain on the total energy consumption for different methods compared to the All-On method where there is no-switching applied.

Fig. 2. Results for total energy consumption of the network and the gain provided by different switching approaches. Note that the results are the averages of 100 runs.

b) All-Off: Opposite to the all-on method, all the SCs are switched off at all the time slots, and their data traffic is offloaded to the MC regardless its available capacity. In other words, sustaining a good level of QoS is at very high-risk in this case, as all the users served by all the SCs are forced to be squeezed in the service of the MC, which results in reducing the QoS for each single users in order to accommodate all the offloaded users as well as the existing ones.

c) Exhaustive Search: Exhaustive search (ES) represents an optimal method in proper CS, such that it attempts to save energy as much as possible by respecting to the constraint of the available capacity of the MC. It tries and checks all the possible switching options, and then find out the best solution, which results in the least power consumption without exceeding the MC capacity. Therefore, it offers gain in power

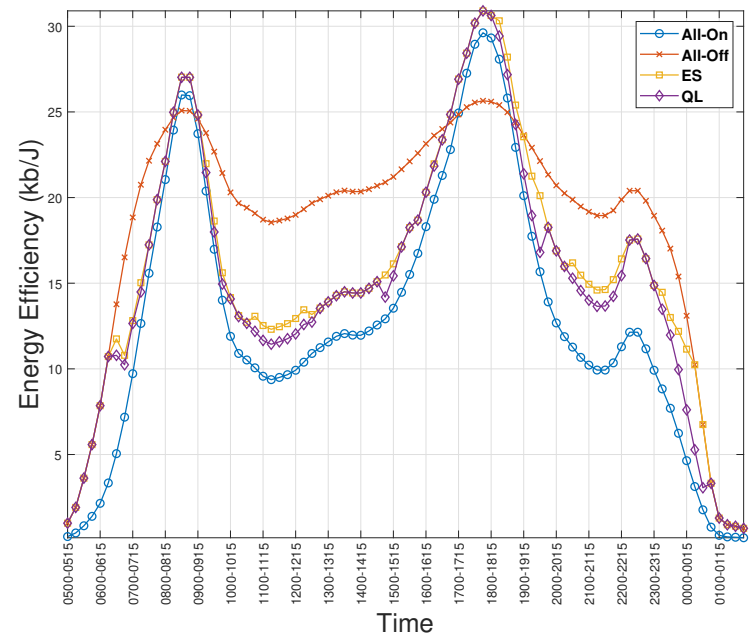

Fig. 3. HetNet energy efficiency.

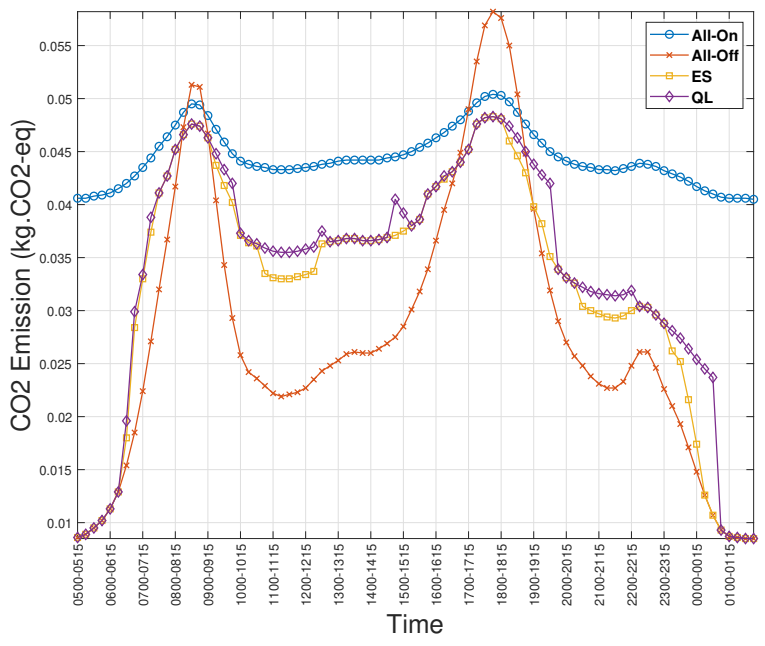

Fig. 4. HetNet $\mathrm{CO}_{2}$ emissions.

consumption and with the reduced $\mathrm{CO}_{2}$ emissions, while maintaining the QoS level for the users.

\section{Metrics}

Three different metrics, namely total energy consumption, percentage gain in the total energy consumption, and the $\mathrm{CO}_{2}$ emission, are used in this work in order to evaluate the performance of the developed CS techniques. First, the total power consumption refers to the accumulated BS energy consumption of the whole network. In other words, the power consumption of the BSs (either MC or SC) are calculated individually and then combined together to obtain the overall power consumption. The calculated power consumptions then multiplied by specific time in which it was achieved to form energy consumption. Second, the percentage gain in the total energy consumption is calculated as follows: 


$$
G(\%)=\frac{E_{\mathrm{on}, \mathrm{t}}-E_{x, \mathrm{t}}}{E_{\mathrm{on}, \mathrm{t}}},
$$

where $E_{\mathrm{on}, \mathrm{t}}$ is the total energy consumption of the All-on method, while $E_{x, \mathrm{t}}$ reflects the total energy consumption of all the other methods provided in Section IV-B, such that $E_{x, \mathrm{t}} \in\left\{E_{\mathrm{off}, \mathrm{t}}, E_{\mathrm{es}, \mathrm{t}}, E_{\mathrm{ql}, \mathrm{t}}\right\}$, where $E_{\mathrm{off}, \mathrm{t}}, E_{\mathrm{ql}, \mathrm{t}}$, and $E_{\mathrm{es}, \mathrm{t}}$ are the total energy consumptions for the All-off, ES, and QL, respectively. Therefore, as it can be inferred from (16) that the All-off, ES, and QL methods are compared to the All-on methods, where all the BSs are kept on, to observe how much energy saving is acquired.

Lastly, to better reflect the energy saving results to establish the $\mathrm{CO}_{2}$ footprint on the environment, this study proposes a process for formulating $\mathrm{CO}_{2}$ emission reduction of overall HetNet architecture when All-On, All-Off, ES and QL methods are envisaged. From (12) the overall impact of $\mathrm{CO}_{2}$ emissions on the area covered by HetNet is calculated where an integral function over a period of time is used.

\section{Results}

Fig. (2a) demonstrates the overall energy consumption of the BSs in the HetNet for all the developed CS methods, while Fig. (2b) presents the gains of All-Off, ES and QL against All-On when no switching is applied. As it can be seen from Fig. (2a) that how much energy can be saved in different time frames of the day against All-On method. When no data is to be carried out through a SC, $P_{\mathrm{sc}}=0$ in (5), then a SC may go into sleep mode with reduced power consumption, hence increasing the gain in Fig. (2b). Fig. (3) demonstrates the overall $\mathrm{EE}$ of the HetNet which is obtained from (6). As it can be seen that All-On method has the worst efficiency compared to All-Off, ES and QL in different times of the day. The novelty of this paper lies in Fig. (4) which determines the overall impact of $\mathrm{CO}_{2}$ footprint across the HetNet environment by comparing All-Off, ES and QL with All-On methods. Carbon emissions have been calculated by using conversion factor as shown in (12). On comparing all methods discussed in this proposal, the overall HetNet energy consumption gain coupled with $\mathrm{CO}_{2}$ between All-On and All-Off is approximately $38.52 \%$, between All-On and ES is approximately $28.21 \%$, and between All-On and QL is approximately $25.2 \%$. From the statistics, we can conclude that the propsoed CS methods would be able to save considerable amount of energy and carbon emissions from the HetNet.

\section{CONCLUSION}

In this article, a concept of energy aware methodology is proposed to analyse the impact of $\mathrm{CO}_{2}$ emissions in a two-tier HetNet by using traffic offloading and CS technique in order to implement smart city planning and green communications agenda. More specifically, $Q$-learning based CS algorithm is used to find an optimal way of under-loaded SCs that should be switched off to improve the overall $E_{\mathrm{HetNet}}$ and $\mathrm{CO}_{2}$. Based on proposed model and simulation results, energy savings gain coupled with carbon emissions of up to $25.2 \%$
TABLE II

SIMULATION PARAMETERS

\begin{tabular}{ll}
\hline Parameter & Value \\
\hline Bandwidth & $20 \mathrm{MHz}$ \\
Frequency & $2.6 \mathrm{GHz}$ \\
Number of RBs per MC & 100 \\
Number of RBs per SC & 100 \\
$P_{\mathrm{max}}^{\mathrm{mc}}, P_{\mathrm{max}}^{\mathrm{sc}}$ & $20 \mathrm{~W}, 6.3 \mathrm{~W}$ \\
$B S_{\mathrm{tot}}^{\mathrm{mc}}, B S_{\mathrm{tot}}^{\mathrm{sc}}$ & $130 \mathrm{~W}, 56 \mathrm{~W}$ \\
$\delta_{\mathrm{mc}}, \delta_{\mathrm{sc}}$ & $4.7,2.6$ \\
Number of iterations & 100 \\
\hline
\end{tabular}

are achieved while ensuring the QoS of users is maintained. We aim to analyse more complexity in our future work where $\mathrm{CS}$ would be used to calculate $\mathrm{CO}_{2}$ emissions by considering user mobility predictions.

\section{REFERENCES}

[1] L. X. N. Z. Oh E., Krishnamachari B., "Toward dynamic energy-efficient operation of cellular network infrastructure." IEEE Communications Magazine, vol. 49, no. 6, pp. 56-61, Jun 2011.

[2] A. M. G. A. Jens Malmodin, Dag Lunden and M. Nilsson, "Life cycle assessment of ict," IEEE Journal of Industrial Ecology, Carbon Footprint and Operational Electricity Use from the Operator, National, and Subscriber Perspective in Sweden., Jun 2014.

[3] Cisco, "Cisco visual networking index: Global mobile data traffic forecast update." Cisco White Paper, Feb 2012.

[4] C.-L. L. H.-L. C. K.-L. H. Chang, Chia-Hsuan and Y.-B. Lin, "A novel lipa scheme for lte voip services with home enbs." IEEE Journal of Wireless Mobile Networks, Ubiquitous Computing, and Dependable Applications, vol. 4, no. 3, pp. 1-22, 2013.

[5] G. Fettweis and E. Zimmermann, "Ict energy consumption - trends and challenges." IEEE Proc. IEEE WPMC, Lapland, Finland, Sept 2008.

[6] A. Mohamed, O. Onireti, M. A. Imran, A. Imran, and R. Tafazolli, "Control-data separation architecture for cellular radio access networks: A survey and outlook." IEEE Article in Comms. Surveys and Tutorials, Jun 2015.

[7] Y.-B. Lin, L.-C. Wang, and P. Lin, "SES: A novel yet simple energy saving scheme for small cells," IEEE Transactions on Vehicular Technology, vol. 66, no. 9, pp. 8347-8356, sep 2017.

[8] S. Zhang, N. Zhang, S. Zhou, J. Gong, Z. Niu, and X. Shen, "Energyaware traffic offloading for green heterogeneous networks," IEEE Journal on Selected Areas in Communications, vol. 34, May 2016.

[9] X. Chen, J. Wu, Y. Cai, H. Zhang, and T. Chen, "Energy-efficiency oriented traffic offloading in wireless networks: A brief survey and a learning approach for heterogeneous cellular networks," IEEE Journal on Selected Areas in Communications, vol. 33, pp. 627-640, Apr 2015.

[10] S. H. Atai Abubakar, Metin Ozturk and M. A. Imran, " $q$-learning assisted energy-aware traffic offloading and cell switching in heterogeneous networks." IEEE Conference paper, 2019.

[11] X. G. M. C. Junliang Ye, Yuanyuan He, "Energy efficiency analysis of $5 \mathrm{~g}$ ultra-dense networks based on random way point mobility models." IEEE International Symposium on (WPMC), 2016.

[12] S. S. I. Hwang, B. Song, "A holistic view on hyper-dense heterogeneous and small cell networks." IEEE Communications Megazine, 2013.

[13] Greenhouse gas reporting: conversion factors 2019 , https://www.gov.uk/government/publications/greenhouse-gas-reportingconversion-factors- 2019.

[14] R. S. Sutton and A. G. Barto, Reinforcement learning: An introduction. MIT press, 2018

[15] G. Auer, V. Giannini, C. Desset, I. Godor, P. Skillermark, M. Olsson, M. Imran, D. Sabella, M. Gonzalez, O. Blume, and A. Fehske, "How much energy is needed to run a wireless network?" IEEE Wireless Communications, Oct 2011. 